\title{
STUDY OF THE EFFECT OF SUSTAINABLE ARCHI- TECTURE ON THE DESIGN OF RESIDENTIAL BUILDINGS (CASE STUDY: QAZVIN PARDIS COMPLEX)
}

\author{
Zeynab MOHAMMADIAN ${ }^{1}$, Majid SHAHBAZ1 ${ }^{1, *}$ \\ ${ }^{1}$ Department of Architecture, Zanjan Branch, Islamic Azad University, Zanjan, Iran. \\ corresponding author: majid.shahbazi254@gmail.com.
}

\begin{abstract}
Housing is a widespread and complex issue with a variety of dimensions. First, housing was considered as a physical location and as a shelter and basic necessity of the households, but today the concept of dwelling as the place that provides all the services and facilities necessary for a better life of the family has been created. One of the issues discussed at many conferences and in general in the scientific community is the definition of sustainability in the psychological point of view, along with the ways to introduce this concept into the architecture and human habitat environments. This research first begins with the main question about how the concept of sustainability can be found within the scope of housing. Then existing resources search is done and accessible projects are analyzed and the weaknesses and strengths of traditional architecture and contemporary architecture are compared. Finally, the relationship between the definitions of three concepts: dwelling, sustainable design and traditional Iranian architecture was discussed. At the end, the sustainable design that is also appropriate to the Iranian culture was presented.
\end{abstract}

\section{Keywords:}

Residence;

Residential complex;

Sustainable architecture;

Weather.

\section{Introduction}

Human beings have been and will be searching for shelter and a safe and relaxing place from the earliest days of their creation. In the past, because the settlements were small, their natural context was comprehensible and the inhabitants were easily linked directly to the surrounding nature [2]. At that time, the settlements created an inseparable part of the order of nature. Nowadays unfair profitable building and depriving man as a being of his particular emotional and mental requirements of privacy and the natural environment, limiting him in tight walls, lack of response to his spiritual needs, improper use of natural energies and environmental degradation have all reduced the quality of human life, especially in the big cities [1]. A sustainable housing approach has been suggested to satisfy humans needs and is an appropriate way to connect with nature and the environment [4]. Also, due to uncontrolled growth of the population and the need to appropriate housing, today the approach to building residential complexes has been considered as an appropriate solution to solve the shortage crisis in housing [5]. According to mentioned facts about sustainability and the need to build residential complexes, investigation the effect of sustainable architecture on the design of residential buildings is important.

We live in a country with the young population and dwelling demand is severely growing, and according to experts' ideas, the construction of residential complexes is a good solution for the exchange of supply and demand in this sector. Today, the construction industry in the country as one of the largest industries in the country, is faced with problems in various fields [3], which is probably based on the lack of attention to research programs and the promotion of research culture in executive and development activities [9]. For example, the design of roads is incompatible with nature, and that creates problems not only for residents but also damages the environmental context [7]. Fortunately, 
today the discussion of sustainable development in architecture is suggested to overcome such problems and provide appropriate solutions [8]. In recent years, demand for dwelling in large residential complexes is increased; the pace of this increase varies depending on the size of the city and its population growth rate.

It is estimated that there are 1.5 million demands for housing units in the country, so if adequate houses are not built to satisfy the needs of the community, "housing crisis" will be created. Of course, it should be kept in mind that, in addition to the quantitative issues, the quality of the demands in various aspects, including cultural and social, must also be noticed because it directly relates to the quality of life, the spirits of the inhabitants. The lack of consideration of those has effects on the private and social life of individuals [6]. Therefore, providing an appropriate house is one of the main concerns of the experts in this sector. Conducting academic research and holding numerous conferences, in order to reach the appropriate models for the construction of residential complex projects, all emphasize the importance of this issue. One of the most important issues in residential complexes is answering this question: How can such projects (design and construction of residential complexes) properly apply sustainability issues and use the advantages of sustainable architecture? Therefore, conducting research projects and recognizing the issues of sustainability and also introducing them into the design process of housing and building architecture to provide an appropriate house for different groups of the community, are the main aims of this research.

\section{Methodology}

\subsection{Method of data collection}

In this research, a method of data collection is a combination of field observations, interviews and analysis of the library resources.

\subsection{Field sampling}

-Visits of the examples that have been built in the country.

- Review of the experts' point of view in urban space design.

- Review of the audience/target group opinions.

- Analysis of the relevant site.

\subsection{Library studies}

- Analysis of the existing samples.

- Collection of information about the culture and customs of the city.

- Geographic location, demographic, economic, cultural, climatic characteristics study.

- Collection of the standards and rules for designing of the different parts.

\subsection{Information analysis method} solution

It is based on the following factors in two stages: basic knowledge and presentation of the

A. Basic knowledge:

$\checkmark$ Problem statement,

$\checkmark$ Understanding the problem,

$\checkmark$ Conclusion.

B. Presentation of the solution:

$\checkmark$ Theoretical foundations,

$\checkmark$ Goal formulation,

$\checkmark$ Formulation of principles and criteria of design,

$\checkmark$ Formulation of designing pattern,

$\checkmark$ Provide a solution,

$\checkmark$ Choosing the optimal presentation of the solution,

$\checkmark$ The final solution. 


\subsection{Knowledge of the study area and the reasons for its selection}

\subsubsection{Geographic features}

Qazvin is the largest city and capital of the Province of Qazvin in Iran. The area of this city is $5693 \mathrm{~km}^{2}$, consisting of 5 counties, 19 sections, 26 towns and 46 villages. Qazvin province is located between the $35^{\circ} 26^{\prime}$ to $36^{\circ} 45^{\prime} \mathrm{N}$ and between $48^{\circ} 44^{\prime}$ to $50^{\circ} 51^{\prime} \mathrm{E}$ and is located in the neighborhood of Gilan province in northern Mazandaran province (NE), Alborz province (E), Markazi Province (in the SE part), Hamedan Province (SW) and Zanjan Province (in the W), Fig. 1. The common language of Qazvin people is Persian.

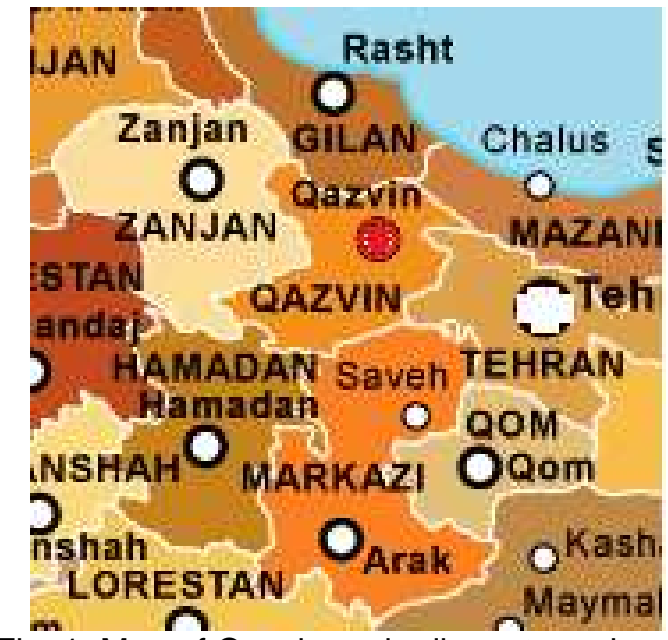

Fig. 1: Map of Qazvin and adjacent provinces.

\subsubsection{Demographic survey of Qazvin Province}

Qazvin Province includes $0.9 \%$ of the area of Iran and $1.7 \%$ of the population of the country with an area of $15,567 \mathrm{~km}^{2}$ and 1273761 inhabitants (2016 census). The population density of Qazvin province is $82 / \mathrm{km}^{2}$ or less than 1 person per hectare, the population density of Qazvin (city) is 118 inhabitants per hectare [8].

\subsubsection{Climatology}

The weather conditions of Qazvin are generally variable - in mountainous areas are conditions depending on the good weather and in plains depending on season. The province is influenced by the western and Mediterranean climate systems that cause the region's rainfall and the northern high pressure system, which is a factor in the cold and humidity of the province. The northern area of Qazvin province is mountainous. This area is cold and has mild winters and mild summers. Plain areas of Qazvin province also have cold winters and hot and dry summers. The average annual temperature is $13.2^{\circ} \mathrm{C}$ and the average annual rainfall is 200 to $300 \mathrm{~mm}$.

\section{Survey of the location of the blocks - urban structure}

\subsection{The hierarchy of the city}

The hierarchy of space, from public and semi-public spaces to semi-public and private spaces in the design of residential complexes is one of the important issues that should be attended. The sequence of the spaces in the context of the traditional settlements of Iran is the relationship of the neighborhood center to the small neighborhood centers with the main passage and to the nonneighborhoods with suburbs. The centers of the neighboring units are reachable through the passageways and they sometimes end in the deadlock / a courtyard. 


\subsubsection{Identity and readability}

Achieving the identity is possible through a variety of measures such as efficiency, maintaining a desirable atmosphere with creating democracy and respecting the human scale, spatial diversity and induction of the possible limits.

\subsubsection{Privacy}

Creating and protecting privacy, preventing direct visions of foreign passers-by to family/ neighborhood private spaces arisen from the cultural and social characteristics of the community and have influenced the design of houses and the establishment of spaces. According to this, in traditional Iranian architecture, the windows of the interior spaces are oriented to the inner courtyard of the house. None of them were opened to the public passage, although they were not at the height of the direct view of passers-by.

\subsection{Analysis and principles in the collection}

In general, a set of residential units or urban spaces that are considered as belonging to the one unit has the following characteristics:

1) They are organized together and combined with each other using specific principle. In other words, a set consists of combining two and more units according to specific characteristics and with a certain definition and order.

2) Proximity, adjacency, continuity or sequence is important features in forming and organizing.

3) The physical or functional program is a distinct, common or complementary component of the collection, such as residential, religious, cultural complexes.

4) Common or similar physical features, such as localization of empty spaces in the plan and the volume, horizontal and vertical axis views.

5) The order and hierarchy in dividing and communicating with each other, connecting the joints of these constituent units and the ways of the connecting units to the rest of settlement urban structure.

6) Common and specific visual quality, such as continuity, harmony, clarity and readability, hierarchy, proportionality and common scales.

\subsection{Analysis of the psychological situation in residential complexes}

Attention to the psychological dimensions of people who live in residential complexes is rather complicated but necessary issue and the aggregation of psychological problems resulting from the restrictions in residential complexes leads to widespread disruptions in personal and social relationships of people that they have lived in these places. Some of the features and conditions that can create these situations are:

1) The lack of a common social class of the inhabitants.

2) Residents' dispute due to minor/irrelevant issues caused by inappropriate architectural design.

3) Lack of adequate light level in the rooms and corridors and visually unattractive views.

4) Lack of attention to the proper implementation of the technical details of the building.

For example: the presence of thin walls of rooms that can transfer the noise of residential units to each other and can cause the problem in these neighbors.

\subsubsection{Factors that create mental disorders and abnormalities in individual behavior}

1) In order to cope with noise transmission, walls of rooms and corridors in buildings should be constructed with noise-resistant materials. In the long term this noise pollution will cause harmful psychological effects, such as weakness of the nervous system, disorder in concentration, mental disturbance and aggression, which can lead to a loss of human performance.

2) Provide adequate and proper light in rooms and corridors

3) Suitable corridors for passage of residents in ordinary and emergency situations, such as transportation of patients.

4) Buildings have visually attractive and suitable appearance and do not use colors that are in contradiction to mental health. 
5) Consideration of the space boundary, which is one of the characteristic of architecture. The benefits of space boundaries are to increase the level of control of individuals over natural stimuli and social interactions.

\subsubsection{The role of designing spaces in comfort of family}

In the continuation of the hierarchy of privacy from the city to the private residential unit, the following points should be applied on the residential units:

1) The separation of public and private spaces of residential units.

2) Providing independence and secrecy to the most private biological spaces.

3) Neighboring the quiet and private spaces of residential units adjacent to each other and away from the noise and traffic.

4) Use of sound resistant structures and materials.

5) Windows and doors and stairs in the vicinity of public spaces should be designed in such a way to provide full security to the residents.

6) Maintaining the independence of the private realm of the residential unit from public spaces, with the proper design of intermediate spaces.

7) The need for open and semi-open space.

8) Try to create direct partnerships of residents with each other.

9) Revival of cultural-historical identities in residential complex.

10) Intervening cultural-historical content in the structure of residential blocks.

\subsubsection{Analysis of the importance of entry in residential complexes}

The entrance in residential complexes should display unique features that are relevant to the site's internal requirements and its human context. The appearance quality of the entrance to the residential collections should also indicate that it is residential. It can be concluded the following paragraph.

1) It is better to create different forms for entrances of the residential complexes. So, the residents, especially children and newcomers will be faced with no orientation problems.

2) Home entrances can be quite varied and diverse. Residents of the home can even be considered as having the right to change or to determine the entrance form.

\subsection{Suggested solutions for incorporating both the objectives of the plan and the factors of the climate}

According to the materials presented in the climate section of the paper, the following ways can be taken in the design of the building:

1) The design of doors and windows should incorporate the new technology of glass production using double-pane aluminium frames and double-pane glazing, equipped with anti-radiation and antishock film, so the heat transfer coefficient of the transparent walls $(k)$ is reduced.

2) Use of the building management system (BMS). Use of smart system in the preparation, transmission and distribution of energy and other consumables based on the half-shutdown of thermal and refrigerating systems in the hours of the day, most of the energy can be saved.

3) Locating less important areas, such as warehouses in such a way that they serve as thermal insulation in walls and cold parts in buildings.

4) Windows fitted with the suitable canopies, which is, in some of the new architectural forms, a semi-transparent and double-glazed pattern fitted on skylight glazing. Canopy is an overhead structure that covers the entire space.

5) Anticipate construction materials with large heat capacity.

6) Use of bright colors on the outer surfaces of the building.

7) Use the balcony to shadow external surfaces.

8) If the design of the walls was not double-sided, the design of the doors should include double-glazed transoms so that the air layer near the ceiling, which is warmer than the rooms, can be cooled down by ventilation and heat exchange.

9) Use of thermal insulations inside and outside the walls depending on the design to reduce the coefficient of heat transfer of the walls $(k)$. 


\section{Introduction of the site}

\subsection{Complete introduction of the site and the reasons for choosing it}

The site is located in the central part of Qazvin city and is well connected by transport to various urban areas. Also, there is easy access to Modarres Boulevard and Khayyam North in the southern part, which are one of the most important shopping streets in Qazvin. In the southern part of the site is also connected to the sport activities. On the north side, through Shahid Lashkari Boulevard, it is connected to two major universities (Imam Khomeini International University and Azad University University). Therefore, it can be said that the site is located in a very favorable place from various aspects. It should be noted that because of the standard access, there is less traffic nearby this site. This site is located on an area that is $6721 \mathrm{~m}^{2}$ with a length $50 \mathrm{~m}$ and width $36 \mathrm{~m}$.

\subsection{Physical characteristics of site}

As it can be seen in the pictures below, the site has access from four sides (Figs. 2 - 4). From the north it is $10 \mathrm{~m}$, east $20 \mathrm{~m}$, west $10 \mathrm{~m}$ and south $12 \mathrm{~m}$. According to the requirements, the pedestrian access is designed from the eastern side of the 20-meter boulevard. The car access is divided to the entrance from the south and exit by the northern side of the site. In general, this area of Qazvin, where the site is located, is designated to provide high-density housing for citizens. That is why this site has been designated for implementation of the project.

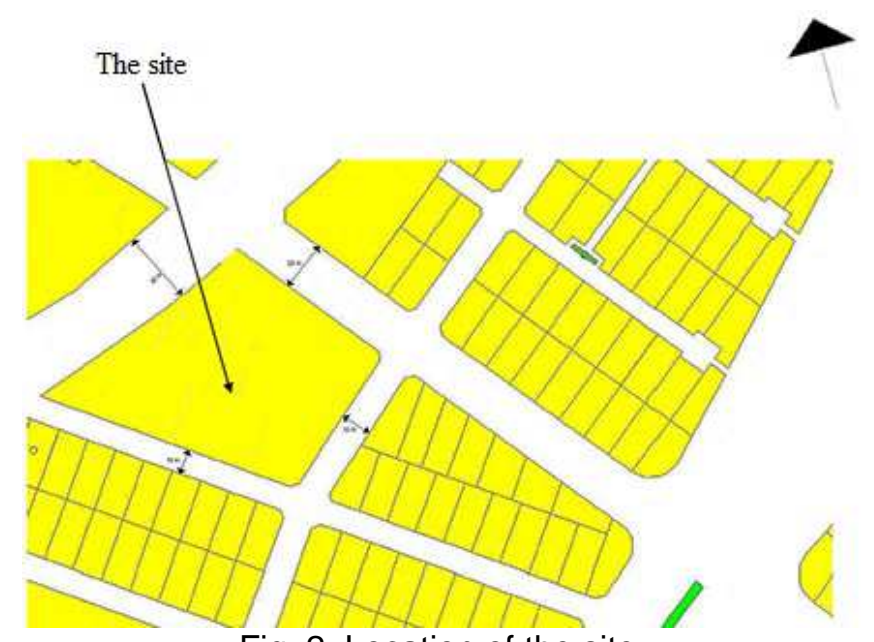

Fig. 2: Location of the site.

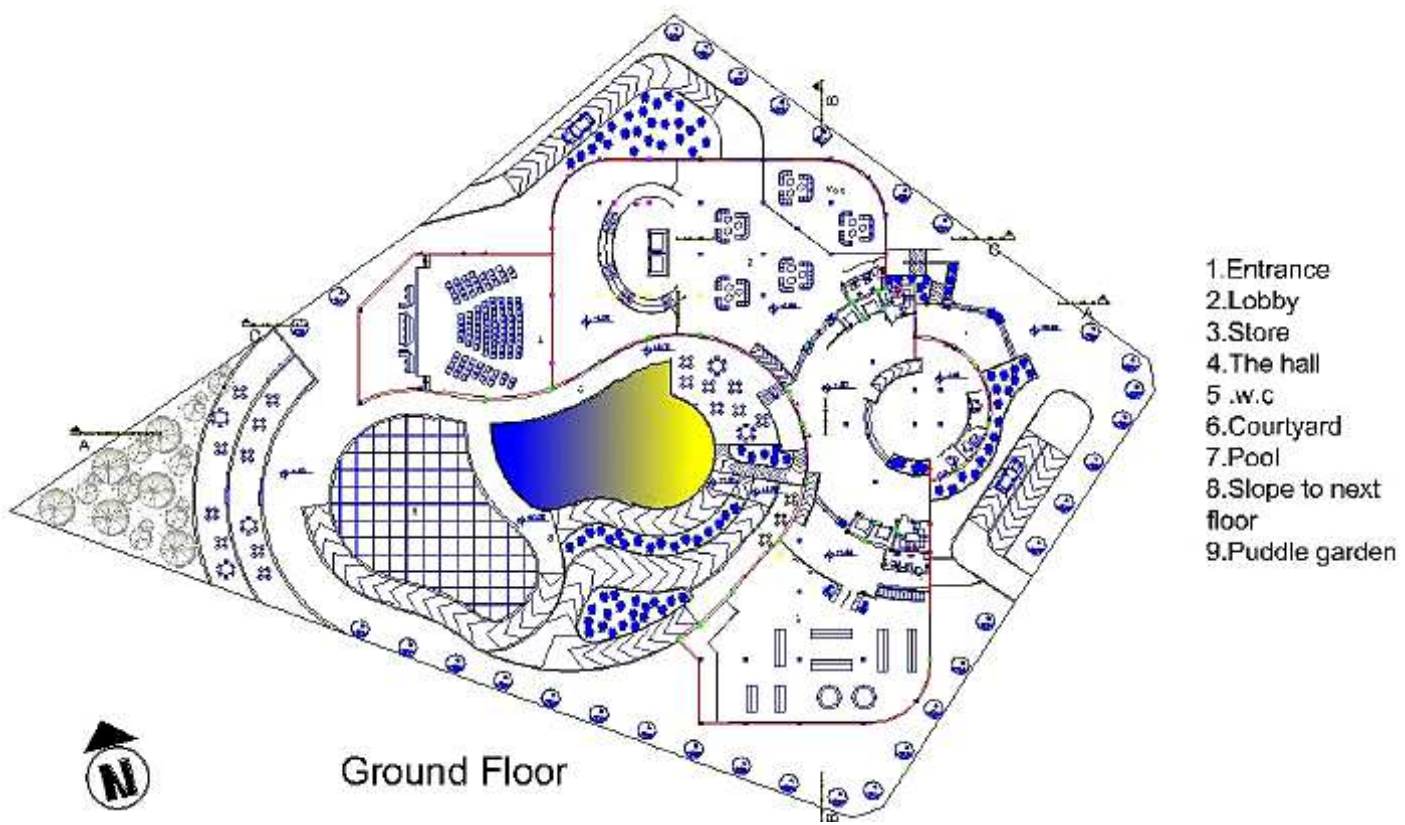

Fig. 3: Ground floor at the site. 


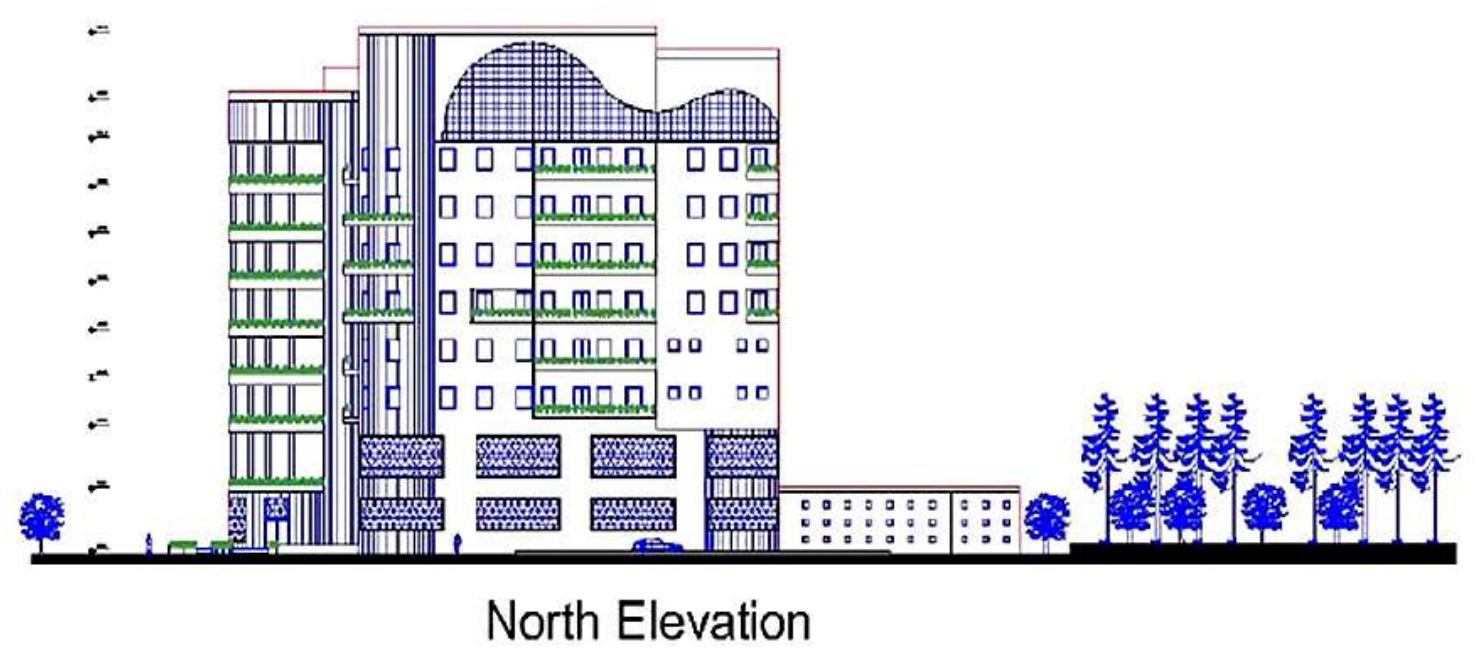

Fig. 4: View of the north side.

\section{Conclusion and ideas}

\subsection{Principles of site design}

1) Optimization for building blocks.

2) Use of plants on the building site and adjacent buildings to get more summer breeze.

3) Use vegetation to shade/cool the area.

4) Use vegetation to control excessive light and undesirable winds.

5) Increase the amount of cooling in the area using evaporation.

6) Increase the reflection of the earth and the outside levels of the windows against the sun in the winter.

7) Use of landforms, adjacent buildings and plants.

8) The use of elements and spaces in the area and the selection of suitable plants, trees and shrubs according to their location on the site.

9) Use a decorative pool for conditioned air.

10) Separation of transport - paths of pedestrians and cars on the site.

\subsection{Conclusion the plan design principles}

1) Reduced area of the external surfaces of walls and roofs.

2) Design and placement of thermal sources to the center of the building.

3) Creating sunlit inner areas to maximize the use of sunlight.

4) Use the vestibule or wind breaker wall at the entrance to the building.

5) Design of secondary spaces such as warehouses, facilities and garages as a reserved space.

6) Entrance and exit of the parking lot in a way that does not create excessive traffic.

7) Use of diversity in the space design to create open, semi-open and closed spaces.

\section{Conclusion}

Architecture as a phenomenon that is created from human thought for the comfort and tranquillity of mankind, and is based on his attitude and intellectual foundations. If we look at this fact fairly, we find that the purpose of architectural creation is not just to respond to physical and material needs and there is a greater purpose, which is to communicate with the deepest emotions and feelings of the human being.

Sustainable design is the design for people and therefore the quality of the interior spaces of the building has special importance. Undoubtedly, desirable quality without attention to nature does not provide adequate lighting and air conditioning for spaces.

In addition, because the sustainability of the building is considered as a goal, therefore, highquality construction and the use of sustainable materials should also be considered. Achieving high 
standards of quality, safety and comfort that actually secures healthy conditions are one of the most important goals of sustainable architecture. Sustainable design is an effort to maximize the comfort of individuals with raising the quality of life and creating the least harm to the surrounding environment. Supply comfort in sustainable design with the least environmental pollution and using natural factors is possible.

\section{References}

[1] AHMADI, F.: Sustainable Architecture, Quarterly Journal of Abadi, Vol. 13, No. 41 - 4, 2003.

[2] AZARBAIJANI, M. - MOFIDI, M.: Concept of Sustainable Architecture. Vol. 1, 5th Edition, 2003, Proceedings of the Conference on Optimizing Fuel Consumption in the Building.

[3] AHARI, Z.: Minimum Housing. 2nd Edition, Building and Housing Research Center, Tehran, 1991.

[4] BAHRAINI, S. H.: Urban Design Process. 2nd Edition, Tehran, Tehran University publication, 2003.

[5] PAYANDEH, A.: Nahj al-Fassah. 4th edition, Tehran, World of Knowledge, 2003.

[6] PIRNIA, M. K.: Introduction to Islamic Architecture of Iran. 7th Edition, Tehran, Iran University of Science and Technology, 1999.

[7] TAVASSOLI, M.: Rules and Criteria for Designing Urban Spaces. 1st Printing, Tehran, Center for Urbanization and Architecture Studies, Tehran, 2000.

[8] HABIBI, A.: Housing Design with Sustainability Approach. Master's Thesis for Architecture, Islamic Azad University, Qazvin branch, 2006.

[9] ELLIOT, J.: An Introduction to Sustainable Development in Developing Countries. 1st Edition, translated by Eftekhari, A. and Rahimi, H., Tehran, Iran Rural Development Institute, 1999. 